\title{
Post-treatment of banknote printing works wastewater ultrafiltration concentrate
}

\author{
Guojun Zhang ${ }^{\mathrm{a}, *}$, Z.Z. Liu ${ }^{\mathrm{b}}$, L.F. Song ${ }^{\mathrm{a}}$, J.Y. Hu ${ }^{\mathrm{a}}$, S.L. Ong ${ }^{\mathrm{a}}$, W.J. Ng \\ ${ }^{a}$ Center for Water Research, Department of Civil Engineering, National University of Singapore, Singapore 119260, Singapore \\ ${ }^{\mathrm{b}}$ Research Center for Eco-Environmental Sciences, Chinese Academy of Sciences, P.O. Box 2871, Beijing 100085, \\ People's Republic of China
}

Received 16 September 2003; received in revised form 5 June 2004; accepted 7 June 2004

\begin{abstract}
A novel process of vortex settling and stage-2 ultrafiltration (UF) with alternating feed direction was used to further concentrate the concentrate produced by a stage-1 UF employed for treatment of banknote printing works wastewater. In this post-treatment process, the final concentrate volume for incineration was reduced by 4-5 times while the permeate of the stage-2 UF could be further reused in the banknote printing operation. It was noted vortex settling facilitated settling of the printing ink and the strategy of regularly alternating feed direction in the UF resulted in a higher permeate flux compared to the corresponding flux for operation without alternating feed direction. The hydraulic retention time (HRT) of the vortex settling tank (VST) used in the pilot-scale experiment was 14 min while feed direction to the stage-2 UF was alternated once every hour. Based on the pilot-scale experimental results, a fullscale system was set up. An economic analysis showed that the novel system was a cost-effective option for posttreatment of stage-1 UF concentrate. The treatment system has been successfully implemented at several Chinese banknote printing companies.
\end{abstract}

(C) 2004 Elsevier Ltd. All rights reserved.

Keywords: Ultrafiltration (UF); Concentrate; Vortex settling tank (VST); Alternating feed direction; Banknote printing works wastewater

\section{Introduction}

Wastewater from banknote printing works mainly contains de-ionized water, sodium hydroxide $(\sim 10 \mathrm{~g} / 1)$, surfactants $(\sim 5 \mathrm{~g} / 1)$ and dispersed printing ink particles, (Parker and Lyons, 1987; Li et al., 1995). Such wastewater is traditionally treated by coagulation and precipitation, and biological methods (Parker and

\footnotetext{
*Corresponding author. Tel.: +65-68746501; fax: +6568745266.

E-mail addresses: cvezgj@nus.edu.sg, zhangguojunmh@yahoo.com (G. Zhang).
}

Lyons, 1987), but a large amount of acid and space is usually needed and treatment results can be unsatisfactory due to the high alkali and printing ink content. Ultrafiltration (UF) is relatively simple and offers many advantages over the traditional process, including no phase changes, no addition of chemicals, ambient temperature operation, and possible recovery of desirable components (Gryta et al., 2001; Miyagi and Nakajima, 2002). Therefore, UF has found increasing use for treating wastewater generated from banknote printing works (Li et al., 1995; Liu et al. 1995). However, there are still two main problems associated with the UF process that need to be overcome, namely membrane 
fouling and cleaning (Zhang and Liu, 2003), and handling of the UF concentrate. A review of the literature revealed that a UF process could remove the printing ink. The resulting permeate, containing sodium hydroxide and surfactants (Turkey red oil), could then be recycled for imprinter application (Li et al., 1995; Liu et al., 1995; Zhang and Liu, 2003). In a batch treatment system, the recovery of permeate could be enhanced by operating the UF at a higher volume concentration ratio (VCR) (Kuo and Chiang, 1987; Kim et al., 2002). However, although the recovery ratio would increase with increasing VCR, the low permeate flux associated with a high VCR inevitably leads to operating difficulties such as a rapid decline in permeate flux and hence shortening of the operating cycle length. In a batch processing plant, the VCR value is therefore usually limited to about 5.0 ( $\mathrm{Li}$ et al., 1995).

Traditionally, direct incineration or coagulation/ incineration has been used for treatment of the UF concentrate (Li et al., 1996). Direct incineration is an expensive process. For example, a particular banknote printing company had incurred an operating cost of about US\$ 610 per day for incinerating $20 \mathrm{~m}^{3}$ of UF concentrate. In order to reduce the volume of UF concentrate (i.e. to reduce incineration cost), coagulation/incineration have also been utilized. In a typical coagulation/incineration system, precipitate generated from coagulation is incinerated while the clarified effluent is further treated at a municipal wastewater treatment plant. The coagulant requirement is, however, large. Furthermore, the clarified effluent would increase the load imposed on the receiving municipal wastewater treatment plant as some residual printing ink will inevitably still be present in the clarified effluent generated by the coagulation process. Aside from the high operating costs, these disposal methods miss the opportunity for recovery of valuable substances (included in the UF concentrate).

In this study, a post-treatment process consisting of vortex settling and stage- 2 UF with alternating feed direction was investigated for further concentrating the stage-1 UF concentrate. The purpose of this posttreatment process was to reduce the total concentrate volume for incineration (i.e. to reduce incineration cost) and to increase overall permeate recovery.

\section{Operating principles}

\subsection{Operating principle of hollow fiber UF with alternating feed direction}

When a hollow fiber UF membrane module fouled by wastewater from banknote printing works was dissected, it was observed the portions of the fibers near the entrance were severely blocked by ink particles. It was

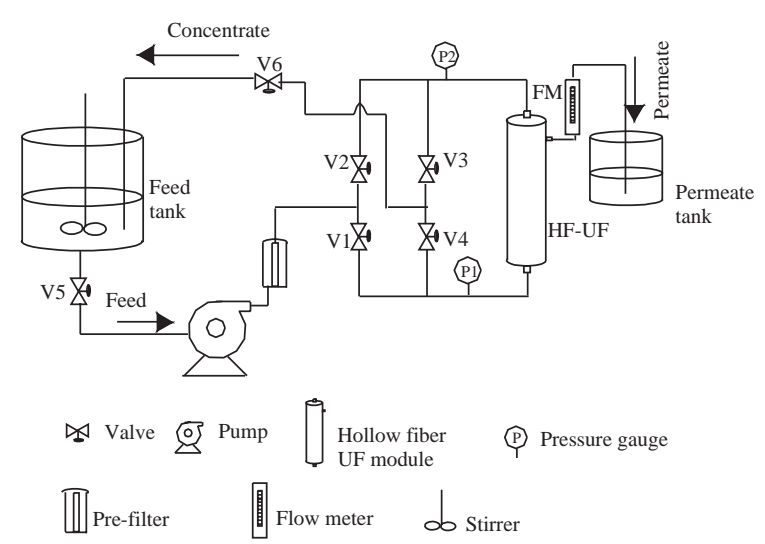

Fig. 1. Schematic diagram of UF system with alternating feed direction.

thought deposition of ink particles at the entrance end of membrane fibers could possibly be reduced if feed direction to the UF module was alternated regularly. This study therefore seeked to develop a UF treatment system in which feed direction could be altered by using a four-pass valve or its equivalent. A schematic diagram of this post-treatment UF system is shown in Fig. 1. As shown in Fig. 1, when V1 and V3 were opened while V2 and V4 were closed, the feed direction was from the bottom to the top. That is, feed was routed through V5, feed pump, and V1. The concentrate was returned to the feed tank through V3 and V6. On the other hand, when V2 and V4 were opened while V1 and V3 were closed, the feed direction was from the top to the bottom.

\subsection{Operating principle of vortex settling tank}

The concentrate produced by stage-1 UF would contain a relatively high concentration of contaminants which may cause operational difficulties to stage-2 UF. The contaminant load to stage-2 UF could be reduced if the inks could be removed before the concentrate was fed into the stage-2 UF modules. Vortex settling exploits centrifugal forces to enhance liquid-solids separation. The centrifugal force was generated by injecting feed into the vortex tank with a tangential velocity. Fig. 2 shows the schematic diagram of vortex settling tank (VST). In the VST, the heavy ink particles would form outer vortices and then precipitate to the bottom while the light clarified solutions would form inner vortices and be directed to the overflow. As shown in Fig. 2, a VST would prolong the liquid flow path which in turn would facilitate settling of printing ink within the vortex tank. Since the stage-1 UF concentrate stream would usually still contain some residual pressures, this could be used to generate the tangential velocity and to lower overall operating costs. 


\section{Material and methods}

\subsection{UF membrane}

The hollow fiber membranes used in this study were produced by the phase transfer method from polysulfone and dichlorodiphenyl sulfone-phenolphthalein condensation polymer $(\mathrm{PS} / \mathrm{PDC} ; \mathrm{MWCO}=30,000)$. The inner diameter of the hollow fiber is $1.1 \mathrm{~mm}$. Ultrafiltration was carried out using cross-flow hollow fiber membrane modules. In the pilot-scale experiments, the UF unit contained four hollow fiber membrane modules with a total effective membrane area of $16 \mathrm{~m}^{2}$.

\subsection{Experimental setup and apparatus}

A schematic diagram of the pilot-scale setup is shown in Fig. 3. Continuous agitation and temperature control were provided in the feed tank (containing stage-1 UF concentrate). The feed, at a predetermined flow rate, was supplied to the alternating feed stage- 2 UF system. The
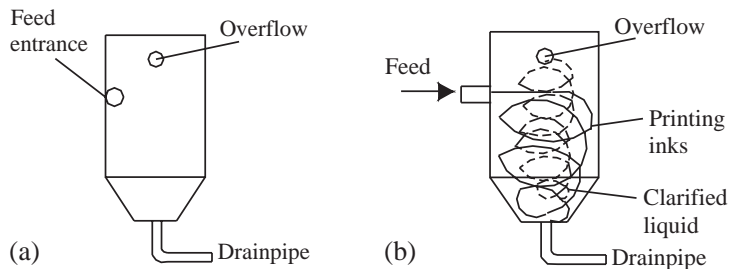

Fig. 2. Schematic diagram of the vortex settler: (a) Basic configuration of vortex settling tank; (b) The process of vortex settling. feed direction was alternated using a four-pass valve as shown in Fig. 3. When the baffle was placed at the a-b position, the feed was fed into the modules from the bottom with the feed routed through V1, pump, prefilter, Point 1, and Point 2. Stage-2 UF concentrate was channeled through Point 4, Point 3 and V3 to the VST. When the baffle was placed at the c-d position, the feed was fed into the modules from the top. A portion of the concentrate could be recycled through V2. The permeate generated was collected in the permeate tank for reuse. It may be noted from Fig. 3 that stage- 2 concentrate was fed into a VST. The clarified solution overflowed the VST into the feed tank for further stage-2 UF treatment. The hydraulic residence time (HRT) in the VST was adjusted using Valves V2 and V3. The diameter and effective volume of the VST were $0.6 \mathrm{~m}$ and 3051 , respectively. A flowmeter was used to measure the permeate flow rates. The pilot-scale experiments were carried out at a banknote printing company.

A schematic diagram of the full-scale system is shown in Fig. 4. The concentrate produced by stage-1 UF was stored in an underground feed tank. The concentrate was lifted by Pump 2 and assisted by Pump 1 and then passed through a cross-flow pre-filter. Pump 3 was the main feed pump. The feed direction could be changed by manipulating the four manual valves (E1, E2, E3 and E4). The stage-2 concentrate was channeled through E4 (or E3) andV6 to the VST. The permeate generated was discharged into a make-up tank and then reused by the printing works. A portion of the permeate was also collected in the cleaning tank. As shown in Fig. 4, stage2 UF concentrate was led into the VST, where the printing ink was settled and then discharged through V1 for incineration. The VST overflow was then returned to

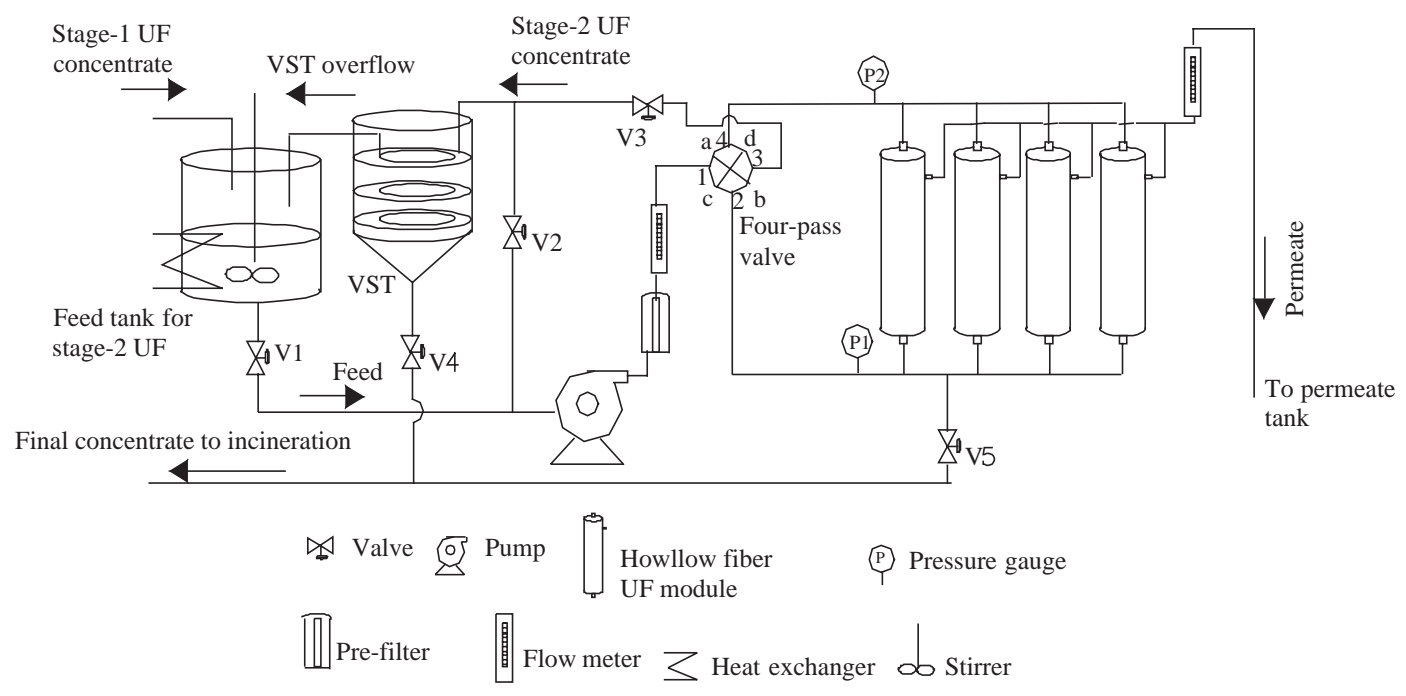

Fig. 3. Schematic diagram of the pilot-scale stage-2 UF and vortex settler system. 


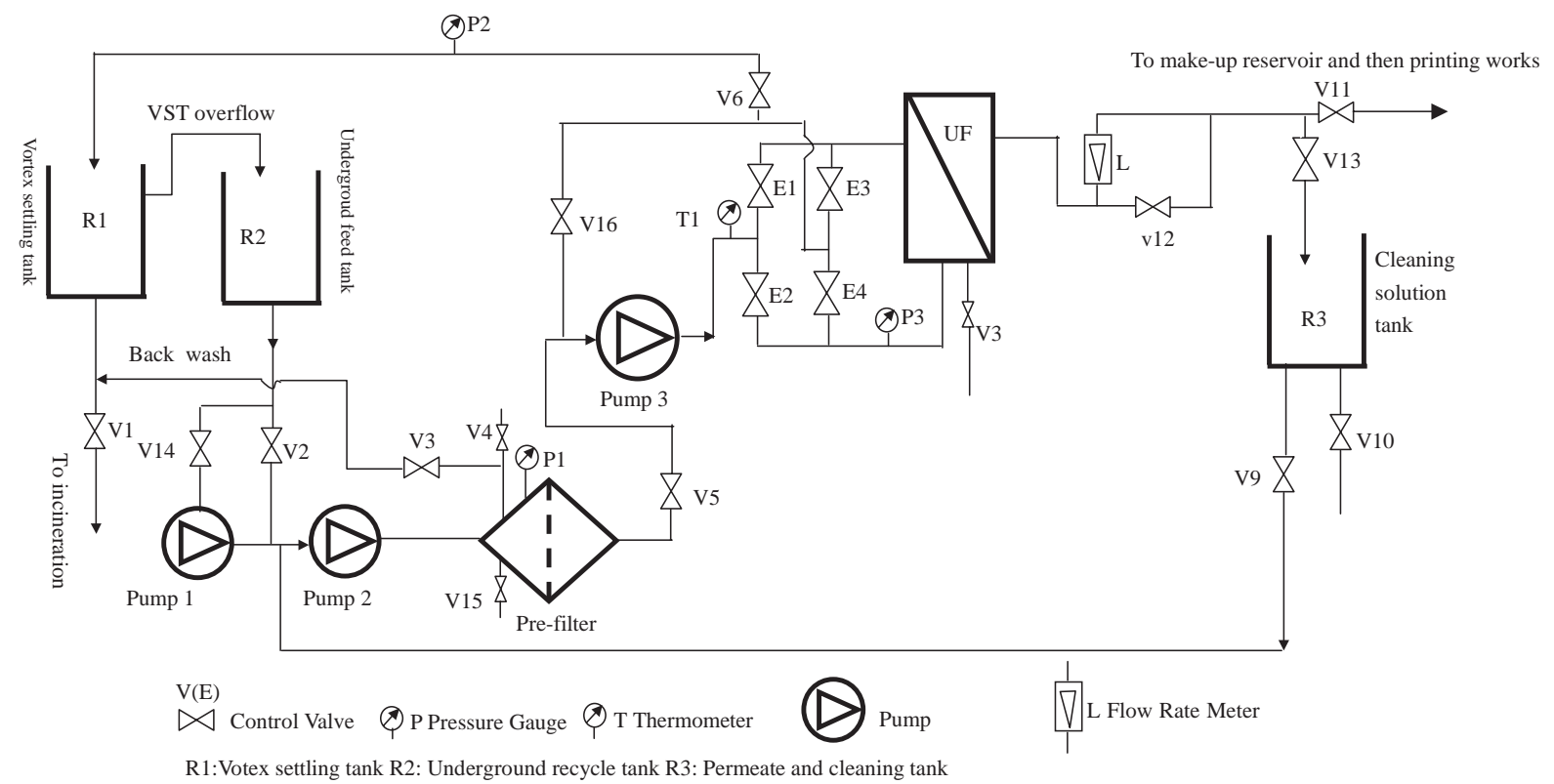

Fig. 4. Schematic diagram of the full-scale stage-2 UF and vortex settler system.

the underground feed tank for further stage-2 UF treatment. The HRT in the VST was adjusted by valves V6 and V16. A backflush pipe, controlled by valve V3, was provided to backwash the vortex tank by operating Pump 2, if large amounts of sludge accumulated at the bottom there.

\subsection{Methods of pilot scale experiments}

Each experiment lasted for about $5 \mathrm{~h}$ treating about $2 \mathrm{~m}^{3}$ of wastewater. UF permeate was used to clean the membrane system at the end of each experiment. VST overflow and bottom concentrate were collected and analyzed regularly. The permeate fluxes were recorded using a flowmeter.

\subsection{Methods of calculation}

In this study, the weight percent of solid content was expressed as follows:

$S(\mathrm{wt} \%)=\frac{W_{\mathrm{a}}}{W_{\mathrm{b}}} \times 100 \%$,

where $S(\mathrm{wt} \%)$ is the solid weight percent of a sample, $W_{\mathrm{a}}$ is the sample weight after drying and $W_{\mathrm{b}}$ is the sample weight before drying. The UF average flux was calculated as follows (Zhang et al., 2003):

$J_{\text {av }}=\frac{V_{\text {total }}}{A t}$,

where $J_{\mathrm{av}}$ is the average permeate flux of membrane modules, $V_{\text {total }}$ is the total permeate volume generated in each experiment, $A$ is the membrane effective area and $t$ is the run time of the experiment.

For each experiment, $\mathrm{VCR}_{1}$ and $\mathrm{VCR}_{2}$ were determined as follows:

$\mathrm{VCR}_{1}=\frac{V_{0}}{V_{0}-V_{\mathrm{p} 1}}$,

$\mathrm{VCR}_{2}=\frac{V_{1}}{V_{1}-V_{\mathrm{p} 2}}$

where $\mathrm{VCR}_{1}$ is the volume concentration ratio of stage-1 $\mathrm{UF}$ and $\mathrm{VCR}_{2}$ is the volume concentration ratio of stage-2 UF. $V_{0}$ is the initial volume (1) of raw wastewater to be treated, and $V_{\mathrm{P} 1}$ is the volume of permeate (l) generated by stage-1 UF process.

$V_{1}$ is the initial volume (1) of stage-1 UF concentrate to be treated, and $V_{\mathrm{P} 2}$ is the volume of permeate (1) generated by stage- 2 UF process.

\section{Results and discussion}

\subsection{Effect of the VST on UF flux}

Fig. 5(a) shows the profiles of VST overflow solids content (wt \%) with time at three different VST HRTs, 7 , 14 and 28 min. As shown in Fig. 5(a), the overflow solids contents (wt \%) at 7 min HRT was significantly higher than the corresponding values associated with 14 and 28 min HRTs. This suggested printing ink could not be adequately separated with a short HRT of $7 \mathrm{~min}$. It was noted from Fig. 5(a) VST overflow solids content (wt \%) 

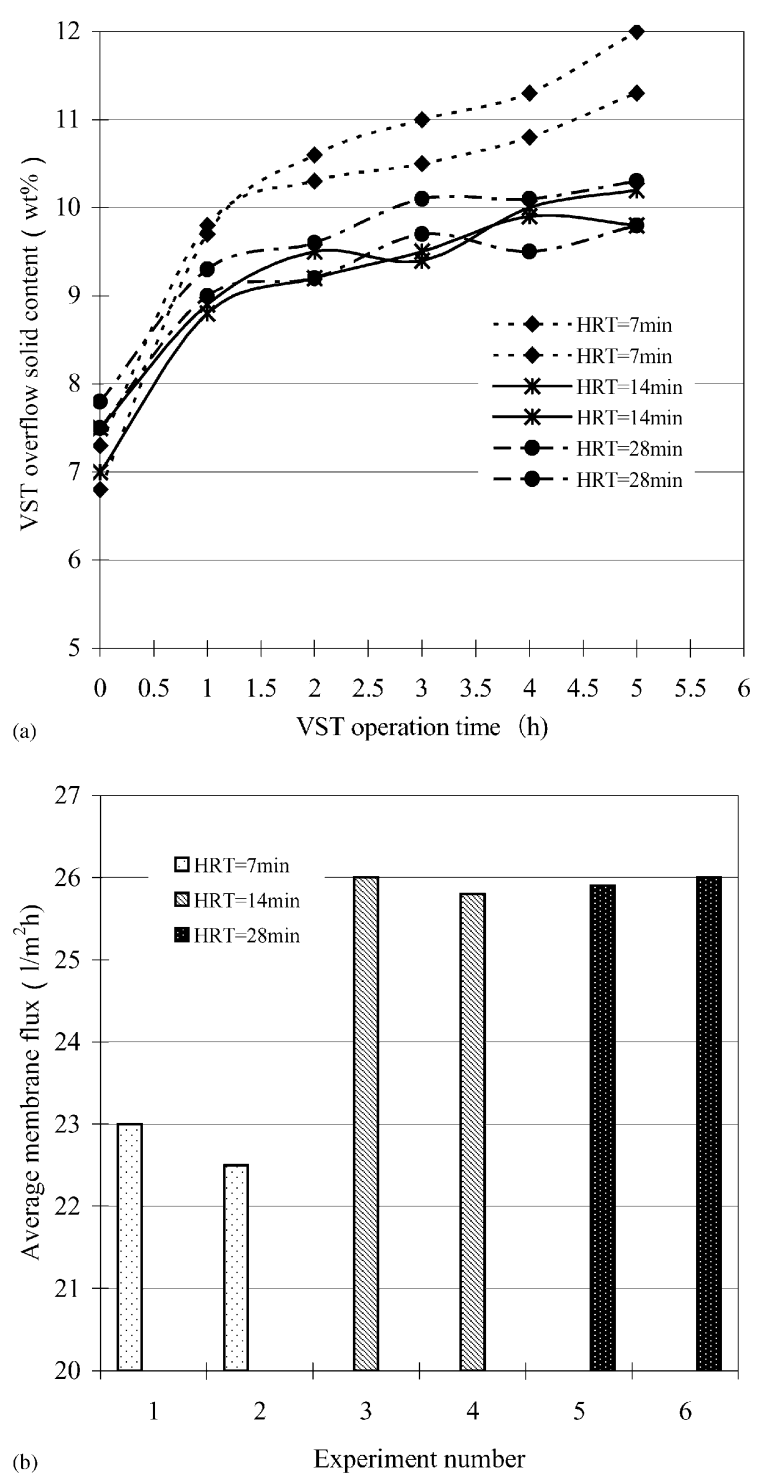

Fig. 5. Effect of the vortex settling on UF flux: (a) The profiles of effluent solid content with time at three different HRTs in the VST; (b) Effects of HRT in VST on membrane average flux $\left(\Delta P=0.12 \pm 0.02 \mathrm{Mpa}, \quad T=45 \pm 2{ }^{\circ} \mathrm{C}, \quad\right.$ circulating velocity $=1.5 \mathrm{~m} / \mathrm{s})$.

at 7 min HRT continued to increase as the experiment progressed. In contrast, the corresponding profiles for 14 and 28 min HRTs indicated a leveling off. It was also noted from the figure the VST overflow solids contents (wt\%) obtained with the 14 and $28 \mathrm{~min}$ HRTs varied within a narrow band. This would suggest the $14 \mathrm{~min}$ HRT was adequate for settling printing inks in stage- 2 UF concentrate.

The effect of VST HRT on average membrane flux is shown in Fig. 5(b). The average membrane fluxes

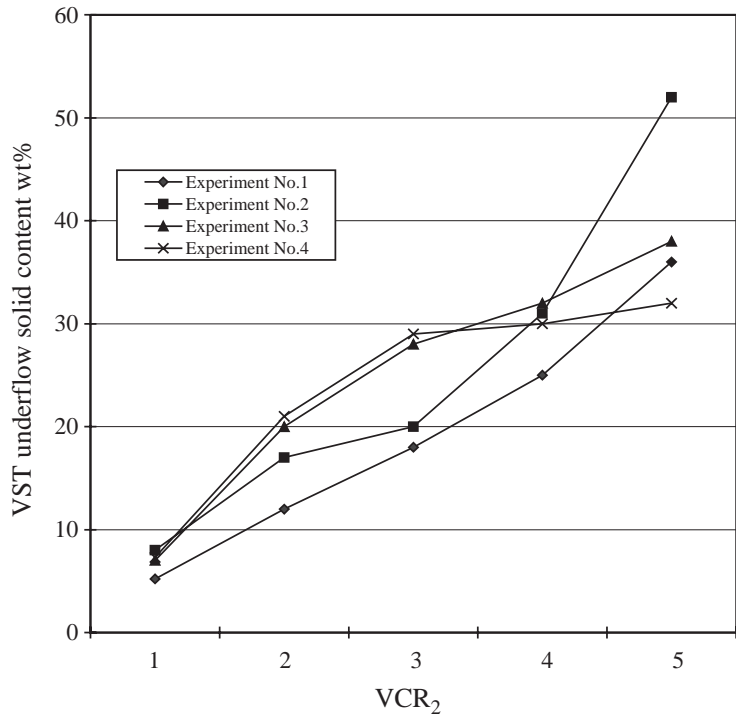

Fig. 6. The relationship between VST solid content ( $\mathrm{wt} \%)$ and $\mathrm{VCR}_{2}$ (HRT of the VST $=14 \mathrm{~min}, \Delta P=0.12 \pm 0.02 \mathrm{Mpa}$, $T=45 \pm 2{ }^{\circ} \mathrm{C}$, circulating velocity $=1.5 \mathrm{~m} / \mathrm{s}$ ).

obtained at $7 \mathrm{~min}$ VST HRT were much lower than those obtained at HRTs of 14 and $28 \mathrm{~min}$. However, the fluxes obtained at 14 and 28 min HRTs were largely similar. The higher VST overflow solids contents (wt \%) associated with 7 min HRT (Fig. 5(a)) led to the much lower average membrane fluxes obtained. These results indicated a $14 \mathrm{~min}$ HRT was sufficient for settling the printing inks. In view of this, 14 min HRT was selected for subsequent experiments.

Fig. 6 shows the relationship between solids content (wt \%) of the VST concentrate collected from the bottom of the vortex tank and the volume concentration ratio $\left(\mathrm{VCR}_{2}\right)$ attained in the stage-2 UF system. It is noted that the VST concentrate solids content increased very rapidly with $\mathrm{VCR}_{2}$ although HRT of the VST was kept at $14 \mathrm{~min}$. Because of variations in the initial solids content batch to batch, these variations resulted in the differences among the four readings obtained for a given $\mathrm{VCR}_{2}$ value. It is noted from Fig. 6 that the solids concentration in the VST concentrate could even exceed $50 \%$. This is very high and may potentially lead to discharge and feed pipe, and feed injection nozzle chocking at the incineration plant.

\subsection{Effect of alternating feed frequency on UF flux}

Fig. 7 shows the effect of the interval between change (i.e. interval between two alternations of feed direction) on membrane flux. Alternating feed direction resulted in higher permeate fluxes. Do note the membrane flux would increase noticeably and sharply immediately following a change in feed direction and hence the two 


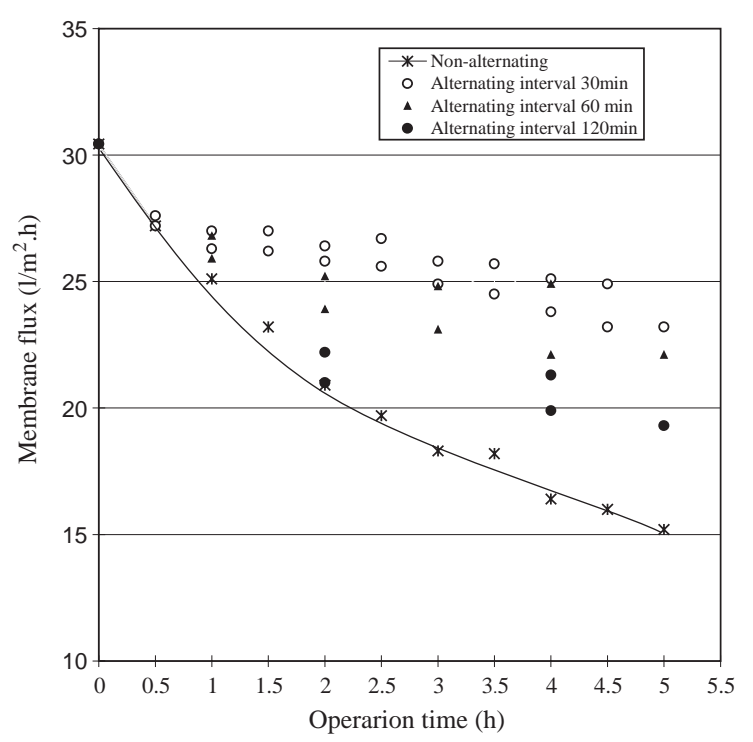

Fig. 7. Effects of exchanging interval on UF flux $\left(\Delta P=0.12 \pm 0.02 \mathrm{Mpa}, \quad T=45 \pm 2{ }^{\circ} \mathrm{C}\right.$, circulating velocity $=1.5 \mathrm{~m} / \mathrm{s}$, the line was the flux without feed direction alternation).

flux values at each point in time on Fig. 7. The advantage arising from the alternating feed operation is obvious. For example, after $5 \mathrm{~h}$ of operation, the $30 \mathrm{~min}$ alternating frequency operation maintained membrane flux at $23.21 / \mathrm{m}^{2} \mathrm{~h}$ compared to $15.2 \mathrm{l} / \mathrm{m}^{2} \mathrm{~h}$ for the non-alternating case.

As outlined earlier, with a single feed direction, ink particles tended to block the membrane fibers at the entrance end. In contrast, incorporation of alternating feed direction enabled the cross flow to wash out ink particles and this alleviated membrane fouling and therefore prolonged lifespan of the membrane modules. A membrane module was dissected at the end of the pilot-scale experiments. It was noted there was no ink intrusion in the membrane fibers at the two entrance ends. Although more frequent feed direction changes enhanced membrane flux maintenance, this would probably stress the equipment. Recognizing this, a 60 min interval was adopted for subsequent study.

\subsection{Effect of the $V C R_{2}$ on $U F$ flux}

Fig. 8 shows the average membrane fluxes obtained from experiments conducted at different values of $\mathrm{VCR}_{2}$. As shown in Fig. 8, average membrane flux decreased with increasing $\mathrm{VCR}_{2}$. A relatively stable average flux of about $23-261 / \mathrm{m}^{2} \mathrm{~h}$ (applied pressur$\mathrm{e}=0.12 \pm 0.02 \mathrm{Mpa}$, temperature $=45 \pm 2{ }^{\circ} \mathrm{C}$, circulating velocity $=1.5 \mathrm{~m} / \mathrm{s}$ ) was obtained when $\mathrm{VCR}_{2}$ was maintained at either 4.0 or 5.0. However, average permeate flux declined seriously when $\mathrm{VCR}_{2}$ was increased to

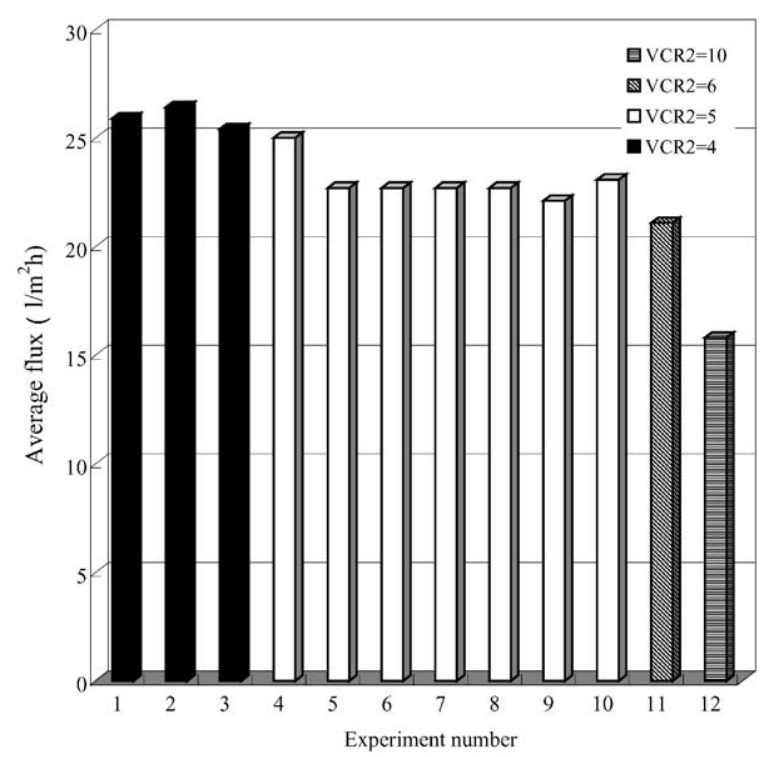

Fig. 8. Effects of $\mathrm{VCR}_{2}$ on UF flux $(\Delta P=0.12 \pm 0.02 \mathrm{Mpa}$, $T=45 \pm 2{ }^{\circ} \mathrm{C}$, circulating velocity $\left.=1.5 \mathrm{~m} / \mathrm{s}\right)$.

10.0. The high feed solid contents associated with a high $\mathrm{VCR}_{2}$ value would lead to serious membrane fouling which in turn rendered permeate flux to decrease significantly. The observation obtained from this series of batch experimental studies suggested that a reasonable $\mathrm{VCR}_{2}$ value for the full-scale system should be within the range of 4.0-5.0.

\subsection{Incineration experiments}

These experiments were intended to examine the time required for incinerating final concentrate, i.e. the mixture of VST bottom concentrate and any residual feed (to stage-2 UF), and to observe if nozzle choking occurred. The results are summarized in Table 1. The time required for incinerating VST concentrate ranged between 32 and $33 \mathrm{~min}$ which was only slight longer than that required for incinerating the same volume of stage-1 UF concentrate. However, the VST concentrate volume requiring incineration was much smaller than the volume of stage-1 UF concentrate. The oil supplement for combusting VST concentrate was only about $27.5-33 \%$ of the requirement for incinerating stage-1 UF concentrate (if stage-1 UF concentrate was incinerated directly). Although the solids content of the final concentrate was very high, no choking was encountered.

\subsection{Operation of the full-scale system}

Based on the pilot-scale results, a full-scale system (Fig. 4) was designed, installed and operated at a banknote printing company. Regarding the actual 
Table 1

Results of incineration experiments

Incineration sample Incineration time(min)

Stage-1 UF concentrate $\left(\mathrm{VCR}_{1}=5\right)$

The final concentrate (the mixture of VST bottom concentrate and the residual feed in the stage-2 UF system) $\left(\mathrm{VCR}_{2}=5\right.$, which is equal to $\left.\mathrm{VCR}_{1}=25\right)$

The quantity of incineration sample was $0.5 \mathrm{~m}^{3}$ every time and the incineration pressure was $16 \mathrm{~atm}$.

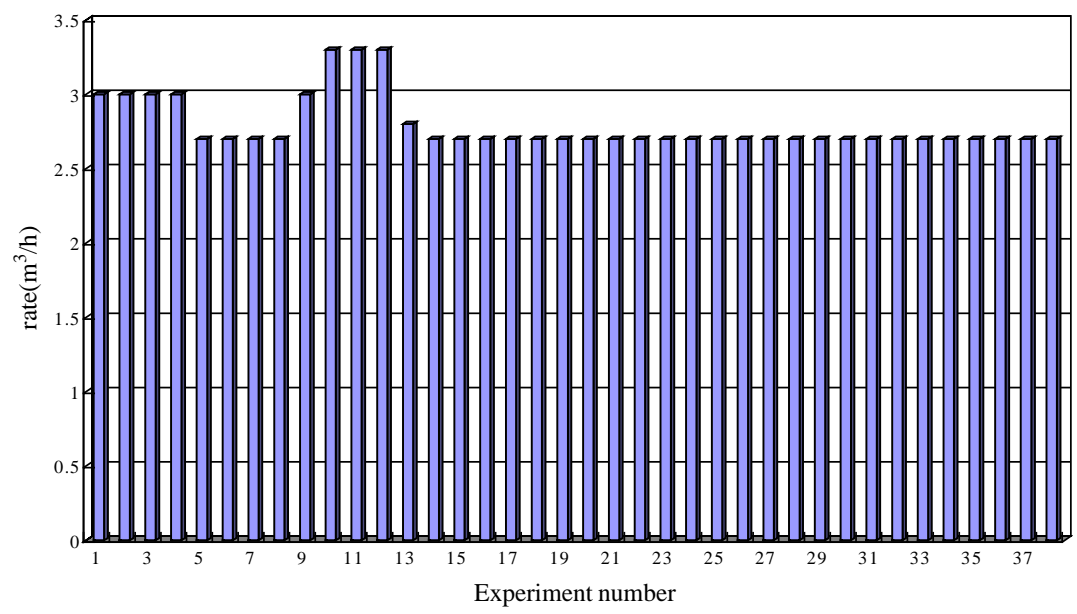

Fig. 9. Plant operation status of industrial-scale stage-2 UF system. The UF membrane area $=24 \times 4 \mathrm{~m}^{2}, \Delta P=0.09 \pm 0.02 \mathrm{MPa}$, circulating velocity $=1.5 \mathrm{~m} / \mathrm{s}, T=45 \pm 2{ }^{\circ} \mathrm{C}, \mathrm{VCR}_{2}=4.0$, the UF permeate was used to clean the modules for about 45 min each day.

conditions at the workshop, two old sediment tanks were reconstructed as the VSTs. The diameter and volume of each VST were 2.0 and $2.0 \mathrm{~m}^{3}$, respectively. The stage-2 UF unit contained 24 hollow fiber membrane modules with a total effective membrane area of $96 \mathrm{~m}^{2}$. Although the design capacity of stage- 2 UF unit was $2.0 \mathrm{~m}^{3} \mathrm{~h}^{-1}$, it was noted that actual loading could reach $2.7 \mathrm{~m}^{3} \mathrm{~h}^{-1}$ (with applied pressur$\mathrm{e}=0.09 \pm 0.02 \mathrm{MPa}$ and temperature $=45 \pm 2{ }^{\circ} \mathrm{C}$ ). The average permeate production rate for 38 experiments is shown in Fig. 9. The system could be operated in a relatively steady manner and about 75 percent of stage- 1 UF concentrate could be further reclaimed for reuse by using stage- 2 UF process and vortex settling.

\subsection{Analyses of economic benefit}

Operating data for the full-scale treatment system and the economic analysis are presented in Table 2. As shown in Table 2, the breakeven on the investment could be achieved after 138 days and the annual economic benefits which could accrue in the first and subsequent years are US\$61,362 and US\$125, 966, respectively. This saving is attractive to the end-user.

\subsection{The revised treatment schematic}

Arising from operating experience with the first fullscale plant, the revised configuration which was used to refurbish plants at other banknote printing works is shown in Fig. 10. About $95 \%$ of the raw wastewater could be reclaimed by using a stage- 1 and stage- 2 UF/ VST system. The final concentrate was incinerated and the incineration residues were disposed off at the factory landfill site. Almost no wastewater was discharged into municipal sewerage system (except for a few cleaning solutions that were used in the cleaning stage).

\section{Conclusions}

A novel post-treatment system involving a VST and stage-2 UF system (with alternating feed direction) for further concentrating the concentrate produced by a stage-1 UF process was found viable for implementation by banknote printing industry for treating wastewater produced by their production operation. It was shown that the strategy of alternating feed direction at regular intervals could achieve a higher permeate flux compared 
Table 2

Basic data and calculations of economic benefits

\begin{tabular}{|c|c|c|c|}
\hline Number & Items & Calculation expressions & Data \\
\hline (1) & $\begin{array}{l}\text { Total quantity of raw wastewater from } \\
\text { banknote printing works }\end{array}$ & & $100 \mathrm{~m}^{3} / \mathrm{d}$ \\
\hline (2) & Stage-1 UF VCR & & 5.0 \\
\hline (3) & Stage-1 UF concentrate & $(1) /(2)$ & $20 \mathrm{~m}^{3} / \mathrm{d}$ \\
\hline (4) & Stage-2 UF VCR 2 & & 4.0 \\
\hline (5) & Final concentrate for incineration & $(3) /(4)$ & $5 \mathrm{~m}^{3} / \mathrm{d}$ \\
\hline (6) & Stage-2 UF permeate & $(3)-(5)$ & $15 \mathrm{~m}^{3} / \mathrm{d}$ \\
\hline (7) & $\begin{array}{l}\text { The value of recoverable materials in } \\
\text { wastewater from banknote printing works }\end{array}$ & & $\mathrm{US} \$ 15.9 / \mathrm{m}^{3}$ \\
\hline (8) & Direct cost of incinerating UF concentrate & & $\mathrm{US} \$ 30.5 / \mathrm{m}^{3}$ \\
\hline (9) & $\begin{array}{l}\text { Pumping costs (based on US\$0.081 per } \\
\text { kw.h)@13KW }\end{array}$ & & US\$1.053/h \\
\hline$(10)$ & Operation time (including the cleaning time) & & $16 \mathrm{~h} / \mathrm{d}$ \\
\hline (11) & Stage-2 UF with VST installation & & US\$82, 927 \\
\hline$(12)$ & $\begin{array}{l}\text { Estimated membrane modules replacement } \\
\text { cost @ } 24 \text { modules } / \mathrm{yr}\end{array}$ & & US\$9756 per year \\
\hline (13) & Operation days/yr & & 240 days/yr \\
\hline (14) & Wages of two operators & & US\$4878/yr \\
\hline (15) & General maintenance & & $\mathrm{US} \$ 2439 / \mathrm{yr}$ \\
\hline$(16)$ & Make-up for recovered material & & US\$1219.5/yr \\
\hline (17) & Stage-2 UF cleaning solutions cost & & $\mathrm{US} \$ 122 / \mathrm{yr}$ \\
\hline (18) & Incineration cost for stage- $1 \mathrm{UF}$ concentrate & $\{(7)+(8)\} \times(3)$ & US\$926.8/d \\
\hline (19) & Incineration cost for the final concentrate & $\{(7)+(8)\} \times(5)$ & US\$231.7/d \\
\hline$(20)$ & Direct economic benefit everyday & $\begin{array}{l}(18)-(19)-(9) \times(10)-\{(12)+(14)+(15)+(16)+(17)\} / \\
(13)\end{array}$ & US\$601.6/d \\
\hline$(21)$ & Breakeven period of the investments & $(11) /(20)$ & 138 days \\
\hline$(22)$ & Financial benefits of the first investing year & $\{(13)-(21)\} \times(22)$ & US $\$ 61,362$ \\
\hline$(23)$ & $\begin{array}{l}\text { Financial benefits of the second investing } \\
\text { year and the succeeding years }\end{array}$ & (13) $(20)-(12)-(14)-(15)-(16)-(17)$ & US $\$ 125,966 / \mathrm{yr}$ \\
\hline
\end{tabular}

Notes: The calculations of incineration cost (Items 18 and 19) included both direct cost of incinerating and the loss value of recoverable materials in wastewater from banknote printing works.

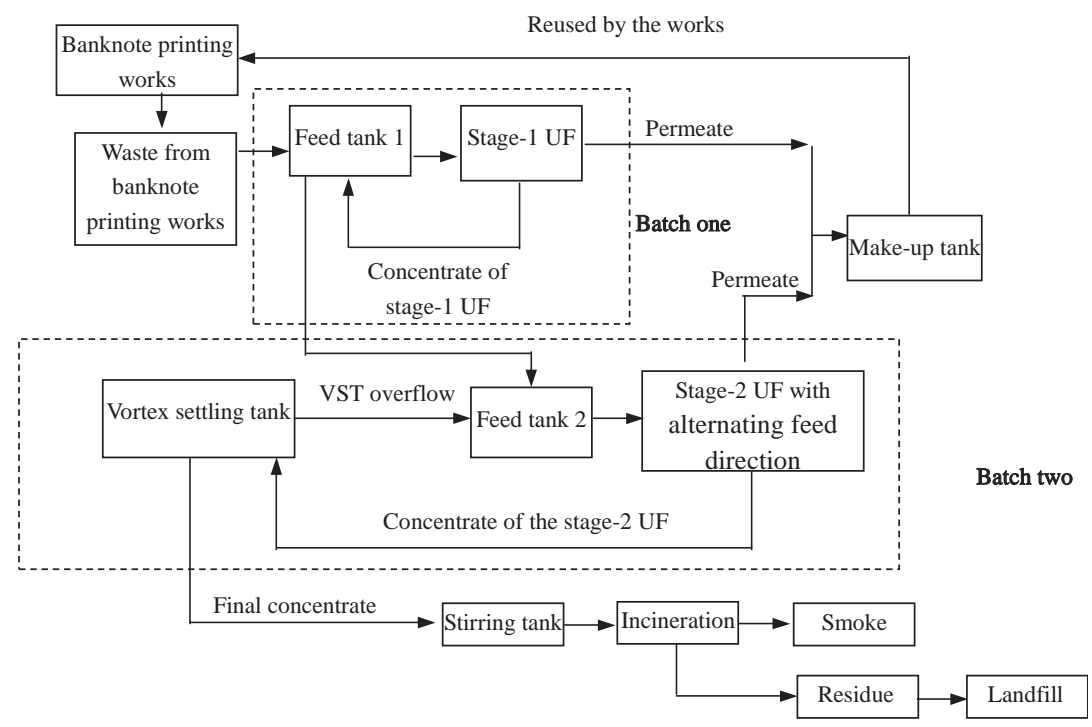

Fig. 10. Schematic diagram of a closed-circuit system for treating wastewater generated from banknote printing works. 
to the corresponding flux for operation without feed direction alternation. Success in maintaining flux was associated with feed direction alternation frequency. The vortex settling can utilize residual energy in the stage-1 UF concentrate and facilitate printing ink separation from the wastewater and in turn reduce the load of stage-2 UF process.

Pilot-scale studies suggested the following design parameters for full-scale plants: VSTs HRT of $14 \mathrm{~min}$; feed direction alternation frequency at once every hour; and the $\mathrm{VCR}_{2}$ at $4-5$ times. In the full-scale system, about $95 \%$ of the raw banknote printing works wastewater could be reclaimed by the stage-1 UF, stage-2 UF and VST system. The final concentrate (about 5 percent of the raw wastewater) was incinerated and the incineration residues were disposed off at a landfill. Consequently, this approach resulted in a zero discharge of wastewater.

An assessment of the economics would suggest such a treatment system is economically viable for posttreatment of stage-1 UF concentrate of banknote printing wastewater. Combined with stage-1 UF, the implementation of this system by banknote printing companies not only reclaimed $95 \%$ of the valuable water for reuse, but also provided savings not achievable with a single-stage UF treatment system.

\section{Acknowledgements}

The authors acknowledge the financial support of the Chinese Academy of Sciences and the National University of Singapore. Appreciation is also extended to Ms. Xiaowei Sun for her contributions in the pilot-scale experiments.

\section{References}

Gryta, M., Karakulski, K., Morawski, A.W., 2001. Purification of oily wastewater by hybrid UF/MD. Water Res. 35 (15), 3665-3669.

Kim, I.C., Kim, J.H., Lee, K.H., Tak, T.M., 2002. Phospholipids separation (degumming) from crude vegetable oil by polyimide ultrafiltration membrane. J. Membr. Sci. 205 (1-2), 113-123.

Kuo, W.S., Chiang, B.H., 1987. Recovery of glutamic acid from fermentation broth by membrane processing. J. Food Sci. 52, 1401-1404.

Li, S.S., Wu, K.F., Guo, X.D., Liu, Z.Z., 1995. Treatment and reuse of wastewater from banknote printing works. In: Proceedings of the Third Intercoastal Academic Workshop on Environmental Protection, Beijing, pp. 107-110 (in Chinese).

Li, S.S., Wu, K.F., Liu, Z.Z., Guo, X.D., 1996. Treatment and reuse methods of wastewater from banknote printing works. Chinese Patent AN96111948 (in Chinese).

Liu, Z.Z., Li, S.S., Wu, K.F., Guo, X.D., 1995. The related effects between membrane and substances of wastewater from banknote printing works, resource, development and environmental protection. In: Proceedings of the Third Intercoastal Academic Workshop on Environmental Protection, Beijing, pp. 131-135 (In Chinese).

Miyagi, A., Nakajima, M., 2002. Membrane process for emulsified waste containing mineral oils and nonionic surfactants (alkylphenolethoxylate). Water Res. 36 (15), 3889-3897.

Parker, J.G., Lyons, B.J., 1987. The dirty dollar and how It's laundered. Water 14 (4), 18-20.

Zhang, G.J., Liu, Z.Z., 2003. Membrane fouling and cleaning in the ultrafiltration of wastewater from banknote printing works. J. Membr. Sci. 211, 235-249.

Zhang, G.J., Liu, Z.Z., Zhao, L., Li, H.B., 2003. Recovery of glumatic acid from ultrafiltration concentrate using diafiltration with isoelectric supernatants. Desalination 154 (1), $17-26$. 